\title{
Phenomenological consequences of a hypothetical light neutral particle in heavy ion collisions
}

\author{
J. Reinhardt, A. Schäfer, and B. Müller \\ Institut für Theoretische Physik, Johann Wolfgang Goethe-Universität, 6000 Frankfurt am Main, Federal Republic of Germany \\ W. Greiner* \\ A. W. Wright Nuclear Structure Laboratory, Yale University, New Haven, Connecticut 06511
}

(Received 17 September 1985)

\begin{abstract}
We discuss the possibility that the line structure observed in the spectrum of the positrons produced in heavy ion collisions is due to the decay of a new neutral elementary particle. We argue that this can be ruled out unless one is willing to accept fine tuning of parameters, or to assume the dominance of nonlinear effects.
\end{abstract}

\section{INTRODUCTION}

Two experimental groups working at Gesellschaft für Schwerionenforschung [GSI (Darmstadt)] have reported the observation of narrow line-shaped structures in the positron spectrum in the collisions of various heavy ion systems with united nuclear charge $Z=Z_{1}+Z_{2}$ in the range $180-188 .^{1-4}$ The effect was found at bombarding energies close to the nuclear Coulomb barrier and appears to be associated with nearly elastic binary scattering events. The energy of the positron line, as determined by the EPOS group, ${ }^{1,3}$ lies between 320 and $360 \mathrm{keV}$ for all systems from $\mathrm{Th}+\mathrm{Th}$ up to $\mathrm{U}+\mathrm{Cm}$. The results from the Orange spectrometer ${ }^{2,4}$ give systematically lower energies around $280 \mathrm{keV}$ for $\mathrm{U}+\mathrm{U}$ and $\mathrm{U}+\mathrm{Th}$ collisions. The linewidth is about $80 \mathrm{keV}$ (or less, taking into account experimental broadening effects) and the production cross section is of the order $\geq 50 \mu \mathrm{b}$. Very recently, indications for positron line structures at about the same energy, although of much reduced intensity, have been reported also for the lighter systems $\mathrm{Th}+\mathrm{Ta}$ (Ref. 5) and $\mathrm{U}+\mathrm{Ta}$ (Ref. 6) ( $Z=163$ and 165).

All attempts to give a conventional explanation of the effect in terms of pair conversion from excited nuclear states have led to contradictions with the experimental evidence. $^{1-6}$ In particular, geometrical and Doppler shift arguments indicate that the emitter system moves with the nuclear center of mass velocity. This would rule out any "background" processes associated with the individual target or projectile nuclei after the collision.

It is tempting to associate the observed line structures with the mechanism of spontaneous positron creation which is expected to occur if the binding energy of the $1 s$ state exceeds twice the electron rest mass. ${ }^{7}$ In ordinary Rutherford scattering this mechanism will not be visible in the shape of the positron spectrum due to the short collision time. ${ }^{8}$ Therefore to explain the positron line structures within this framework ${ }^{9}$ it is necessary to assume the occurrence of time-delayed nuclear reactions. This mechanism will enhance spontaneous positron production, as had been anticipated earlier. ${ }^{10} \mathrm{~A}$ quantum mechanical description has been given ${ }^{11}$ based on the concept of a pocket in the internuclear potential ${ }^{12}$ or in the framework compound nucleus theory. ${ }^{3}$

Although faced with several questions from the nuclear physics point of view, the formation of long-lived "giant" nuclear systems can explain many of the observed features of the positron spectra. One severe difficulty, however, is the near constancy of the line energy observed in the experiments with different projectile and target combinations (keeping in mind, however, that at present the two sets of experimental data do not lead to the same energy). The $1 s$-binding energy is expected to vary strongly with total nuclear charge $Z_{1}+Z_{2}$, other parameters such as the shape of the nuclear charge distribution being equal, i.e., if the nuclear radii scale as expected from conventional nuclides.

Looking at the experimental spectra one might be led to the conclusion, that all the observed positron lines have a single common origin. One mechanism which in a natural way would lead to exactly the same positron energy in all systems is the creation of a new light particle $X$ decaying under monochromatic positron emission. ${ }^{14,15} \mathrm{Al}$ though such an explanation is highly speculative it seems worthwhile to investigate its consequences and check its consistency with the experimental facts. In this paper we want to elaborate on the arguments presented in Ref. 14.

We will concentrate on the hypothesis that the new particle is a neutral boson decaying into an electron-positron pair, $X \rightarrow \mathrm{e}^{+}+e^{-}$. Assuming decay at rest, the mass required to explain a positron energy of $E_{\mathrm{e}^{+}}=330 \mathrm{keV}$ is $m_{X}=2\left(m_{\mathrm{e}}+E_{\mathrm{e}^{+}}\right) \simeq 1.68 \mathrm{MeV}$. Alternative explanations would require the new particle to be charged so that it would be hard to explain why it has not been detected earlier in other experiments. Furthermore, current theoretical models of elementary particle physics accommodate most easily a light neutral boson.

We want to make clear from the beginning that our analysis is not applicable to nonlinear phenomena. As it is impossible to analyze the consequences of all conceivable nonlinear models we omit them totally from this discussion. 
One candidate for the new particle is the pseudoscalar axion which has been postulated on theoretical grounds in order to explain the absence of $C P$ violating terms in the theory of strong interaction. The standard axion model of Peccei and Quinn ${ }^{16,17}$ makes definite predictions on the mass and various coupling constants in terms of only one unknown parameter. This standard axion model is in severe conflict with the experiments, e.g., on the $J / \psi$ and $\Upsilon$ decay, ${ }^{18}$ but it is conceivable that a modified scheme could avoid these contradictions. In view of these uncertainties we will avoid basing our analysis on any specific model, and leave open the type of coupling of the particle.

Sections II to IV of this paper are devoted to the kinematical aspects of the two-body decay $X \rightarrow \mathrm{e}^{+}+\mathrm{e}^{-}$. The narrowness of the positron line structure to be explained will pose certain requirements on the particle creation mechanism. Subsequently we consider the coupling of the new particle to the leptons. Its influence on the electron (and muon) anomalous magnetic moment is calculated and we deduce upper limits on the $X$-e- and $X$ - $\mu$-coupling constants from the present level of agreement between experiment and theory. Using this constraint, an estimate of the production cross section via coupling to the atomic electrons in heavy ion collisions falls short of the observed positron line intensity by several orders of magnitude. In Sec. $\mathrm{V}$ of this paper a similar analysis of high-precision atomic data (e.g., the Lamb shift in hydrogen) is used to narrow down the possible range of the coupling constant to the nucleus.

Subsequently an explicit calculation of nuclear bremsstrahlung of scalar and pseudoscalar particles in slow heavy ion collisions is presented. It is shown in Sec. VI that such a mechanism can explain neither the shape nor the intensity of the positron line structures. Finally we present some considerations on the role of light bosons as resonances in low energy electron-positron collisions.

\section{THE SHAPE OF THE POSITRON SPECTRUM}

Let us discuss the kinematical aspects of the hypothesis that in heavy ion collisions new particles of mass $m_{X}$ are created which decay into electron-positron pairs. The resulting energy distribution of positrons (or electrons) would be monochromatic, with kinetic energy $E_{\text {kin }}$ $=\frac{1}{2} m_{X}-m_{\mathrm{e}}$, only if the particles were created at rest in the laboratory frame. In a more realistic situation the spectrum will be broadened due to several facts: (1) the distribution of the created bosons will have a finite energy spread in the heavy ion center-of-mass system. (2) The proper momentum of the moving particles adds up to the momentum of the decay products depending on a varying relative angle. (3) An additional Doppler broadening will arise from the transformation from the center-of-mass frame to the laboratory frame.

Therefore the mere fact that particles with an appropriate mass $m_{X}$ are created does not automatically guarantee the emergence of a line in the positron spectrum having the observed ${ }^{1-4}$ narrow width $\Gamma \leq 80 \mathrm{keV}$. The actually observed shape of the positron spectrum will depend on the production mechanism of the new particle and also on the detector geometry. Let us assume for simplicity that the production, looked at from the heavy ion center-ofmass frame, is isotropic. If also the two-body decay of the $X$ particle is isotropic (which is obvious for spin-0 particles), a simple expression for the spectrum of positrons applies, ${ }^{19}$

$\frac{d w}{d E^{\prime}}=m_{X}\left(m_{X}^{2}-4 m_{\mathrm{e}}^{2}\right)^{-1 / 2} \int_{E_{-}}^{E_{+}} d E_{X}\left(E_{X}^{2}-m_{X}^{2}\right)^{-1 / 2} \frac{d w}{d E_{X}}$,

where

$E_{ \pm}=\left(m_{X} / 2 m_{\mathrm{e}}^{2}\right)\left[E^{\prime} m_{X} \pm\left(E^{\prime 2}-m_{\mathrm{e}}^{2}\right)^{1 / 2}\left(m_{X}^{2}-4 m_{\mathrm{e}}^{2}\right)^{1 / 2}\right]$.

$E^{\prime}$ is the c.m. positron energy. In the case of a monoenergetic source the integral in Eq. (1) would lead to a boxshaped positron spectrum of a width

$$
\Delta E=\left(E_{X}^{2}-m_{X}^{2}\right)^{1 / 2}\left(m_{X}^{2}-4 m_{\mathrm{e}}^{2}\right)^{1 / 2} / m_{X}
$$

centered at $E^{\prime}=\frac{1}{2} E_{X}$.

Going from the c.m. to the laboratory frame entails an additional Doppler broadening. The transformation relation is

$$
\frac{d w}{d E}=\frac{1}{2} \int d \cos \theta \frac{p}{p^{\prime}} \frac{d w}{d E^{\prime}},
$$

where the energies $E$ and $E^{\prime}$ are related by $E^{\prime}$ $=\gamma(E-\beta p \cos \theta)$ where $\gamma=\left(1-\beta^{2}\right)^{-1 / 2}$ and $\beta=v_{\text {c.m. }} / c$.

If the particles are radiated dynamically during the course of the heavy ion collision, their energy distribution $d w / d E_{X}$ must be rather broad, reflecting the available "Fourier frequencies" of the nuclear motion or, put differently, the time-energy uncertainty relation. At energies close to the Coulomb barrier the characteristic, unavoidable energy spread is of the order of several hundred $\mathrm{keV}$. This essentially is true for nuclear and atomic Coulomb excitation and for $\mathrm{x}$-ray emission processes.

As it turns out, the possible emergence of a line structure in the spectrum of the decay products depends sensitively not only on the falloff constant of the particle spectrum but even more so on its behavior at small kinetic energies. This is readily understood from Eq. (1) since the decay of fast particles leads to a large motional Doppler broadening.

To investigate this point in more detail let us calculate $d w / d E$ explicitly. As the proposed particle production mechanism is not well understood we will discuss three specific models for the particle energy distribution $d w / d E_{X}$.

Guided by the shape of $\delta$-electron spectra one may approximate the particle energy spectrum by a simple exponentially decaying function

$$
\frac{d w}{d E_{X}} \simeq \frac{1}{\Gamma} e^{-\left(E_{X}-m_{X}\right) / \Gamma}
$$

The resulting positron spectrum is shown in Fig. 1 for three values of the falloff constant $\Gamma=100,300$, and 1000 $\mathrm{keV}$. We have assumed a mass $m_{X}=1.68 \mathrm{MeV}$. The half-widths of the curves range between 250 and $700 \mathrm{keV}$. Thus even for the extreme assumption $\Gamma=100 \mathrm{keV}$ the linewidth comes nowhere near the value $\Gamma_{\mathrm{e}^{+}}^{\text {expt }}$ required by 


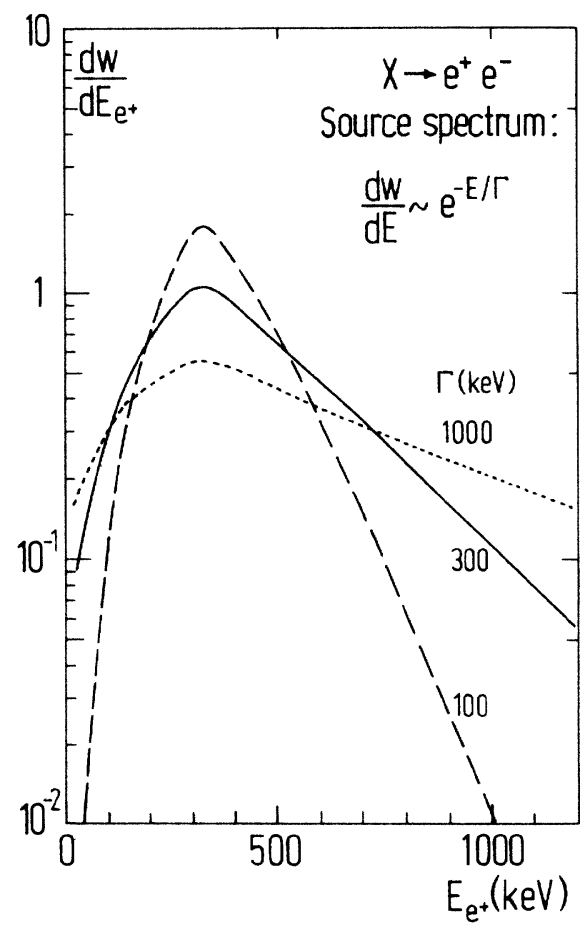

FIG. 1. Positron spectrum resulting from the decay $X \rightarrow \mathrm{e}^{+} \mathrm{e}^{-}$, if the energy spectrum assumed for the $X$ particles is of the form $d w / d E_{X} \simeq 1 / \Gamma \exp \left[-\left(E_{X}-m_{X}\right) / \Gamma\right]$. Three different values of the falloff constant $\Gamma$ have been assumed.

the experiments.

As already discussed qualitatively this situation would change if the low-velocity component of the particle spectrum was enhanced. This can be described, e.g., by making an ansatz similar to (3) for the momentum distribution

$$
\frac{d w}{d p_{X}} \simeq \frac{1}{\Gamma} e^{-p_{X} / \Gamma}
$$

The expression (4) differs from (3) mainly through the factor $d p_{X} / d E_{X}=E_{X} / p_{X}$ which heavy weights the low momentum part of the spectrum. The resulting positron spectra, shown in Fig. 2, are $70-130 \mathrm{keV}$ wide, taking the same values for $\Gamma$ as above. The curves have a flattened maximum due to the Doppler broadening of the c.m. motion. In a given experiment this effect will depend on the detector geometry.

With the ansatz (4) sufficiently narrow positron lines can be produced for reasonable values of the falloff constant $\Gamma$. However, the enhancement of low momentum emission seems not to be justified by models for the particle creation process. On the contrary, in the bremsstrahlung calculation discussed in Sec. VI the spectrum is depressed at small momenta. If we choose, motivated by this model,

$$
\frac{d w}{d E_{X}} \simeq\left(p_{X} / E_{X}\right)^{5} e^{-\left(E_{X}-m_{X}\right) / \Gamma}
$$

with $\Gamma=380 \mathrm{keV}$ the resulting positron spectrum is extremely flat, cf. Fig. 3 (upper curve).

To complete the discussion of energy distributions, a further effect has to be mentioned which can lead to a

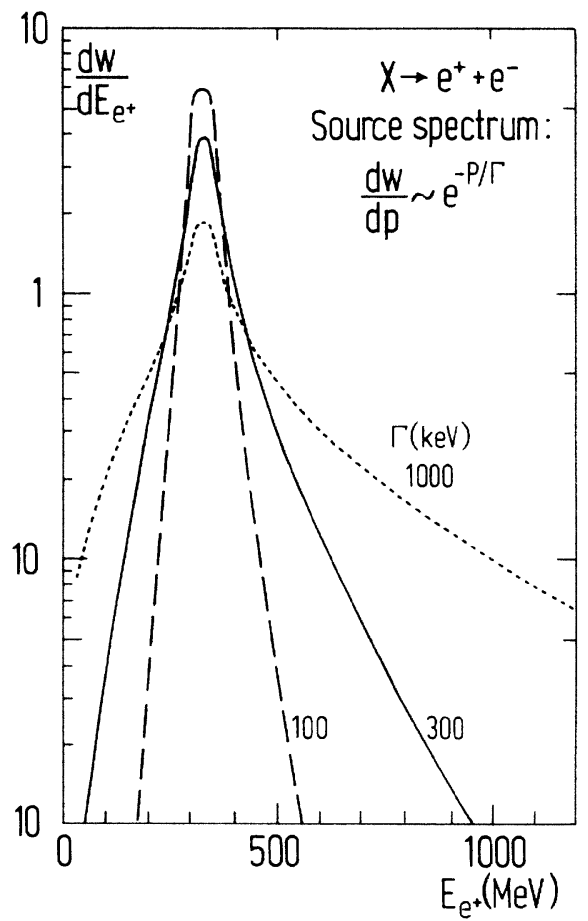

FIG. 2. Same as Fig. 1 if the spectrum assumed for the $X$ particles is of the form $d w / d p_{X} \simeq 1 / \Gamma \exp \left(-p_{X} / \Gamma\right)$.

narrow positron line: If the lifetime $\tau$ of the $X$ particle is comparable to the time needed to leave the finite sensitive volume of the detector then mainly the decay products of slow particles will be detected. This again will enhance the central region of the energy spectrum compared with

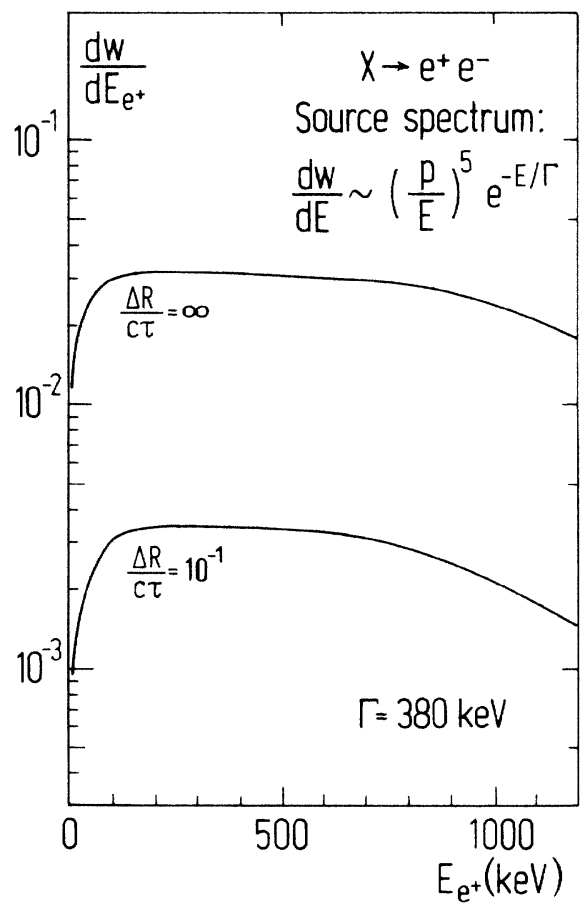

FIG. 3. Same as Fig. 1 if the spectrum assumed for the $X$ particles is of the form

$d w / d E_{X} \simeq\left(p_{X} / E_{X}\right)^{5} \exp \left[-\left(E_{X}-m_{X}\right) / \Gamma\right]$. 


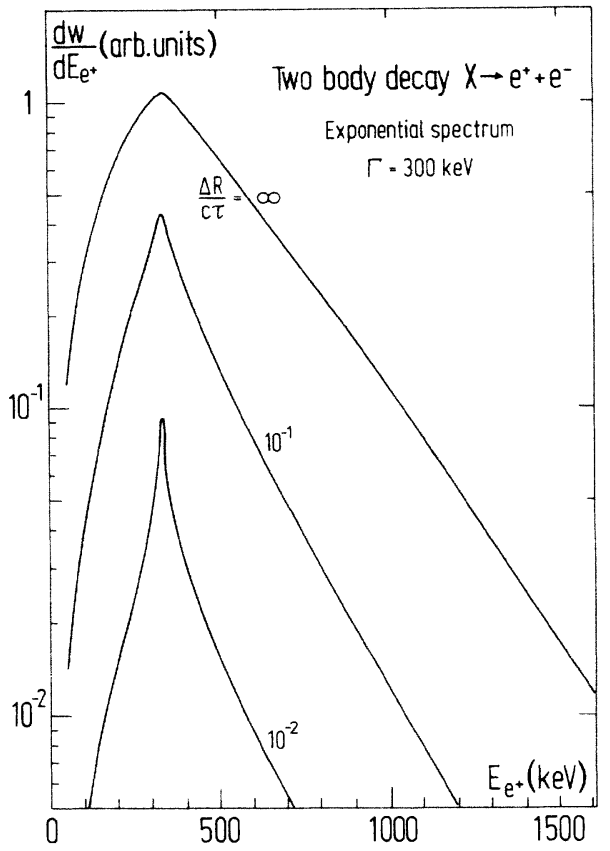

FIG. 4. Positron spectrum as in Fig. 1, assuming $\Gamma=300$ $\mathrm{keV}$. The two lower curves demonstrate the effect of a finite lifetime allowing the escape of fast $X$ particles out of the sensitive region of the detector. Two values of the ratio $\Delta R / \tau$ have been assumed, cf. Eq. (6).

the wings. Neglecting any details of the experimental setup, the effect can be taken into account by multiplying the particle spectrum with the (velocity dependent) decay probability

$$
P\left(E_{X}\right)=1-\exp \left(-\Delta R m_{X} / \tau p_{X}\right),
$$

where $\Delta R$ is a measure for the extension of the sensitive region of the detector. Since this condition has to be imposed in the laboratory frame and not in the center of mass system, we have found it convenient to determine the positron spectrum by Monte Carlo sampling. Figure 4 demonstrates that the finite lifetime argument applied to the case of ansatz (3) indeed can be used to obtain a sufficiently narrow positron spectrum. Assuming a falloff constant $\Gamma=300 \mathrm{keV}$ the parameter $\Delta R / c \tau$ was chosen as $\infty$ (as in Fig. 1), 0.1 , and 0.01 (this corresponds to lifetimes of $0 \mathrm{~s}, 10^{-9} \mathrm{~s}$, and $10^{-8} \mathrm{~s}$, if $\Delta R \simeq 3 \mathrm{~cm}$ ).

However, as can be seen, the narrow linewidth in Fig. 4 is bought at the expense of emission intensity since now most of the particles decay outside the sensitive region of the detector. Furthermore, since the mechanism depends on the presence of a low-momentum component in the particle spectrum, it will not work for the ansatz of Eq. (5) and similar models. This is demonstrated by the lower curve in Fig. 3 where $\Delta R / \tau=0.1$ was assumed.

\section{THE ANOMALOUS MAGNETIC MOMENT}

The existence of a light particle coupling to leptons will make itself felt not only if it is created on mass shell but also through virtual processes, i.e., vacuum fluctuation effects. Most notably, the $g$ factor of the electron (and muon) will be affected. Since these values are known experimentally, and understood theoretically, to an exceedingly high accuracy, this will lead us to stringent upper limits for the $X$-particle-lepton coupling constant $g_{i}^{\mathrm{e}}$ (and $\left.g_{i}^{\mu}\right)$.

The main contribution to the anomalous magnetic moment of a Dirac particle is determined by the first-order radiative correction to the photon-lepton vertex [Fig. $5(a)$. In the notation of Ref. 20 the vertex function is given by

$$
\Lambda_{\mu}\left(p^{\prime}, p\right)=-\left(\alpha_{i}^{\mathrm{e}} / 4 \pi\right) \int d z_{1} d z_{2} d z_{3} \delta\left(1-z_{1}-z_{2}-z_{3}\right) G_{\mu}\left(p^{\prime}, p, z_{k}\right) /\left[m^{2}\left(1-z_{1}\right)^{2}+m_{X} z_{1}-q^{2} z_{2} z_{3}\right]
$$

where we have abbreviated the matrix function

$$
\operatorname{ei}^{g^{e} \Gamma\left\{\frac{i}{g^{e} \Gamma e}\right.}
$$

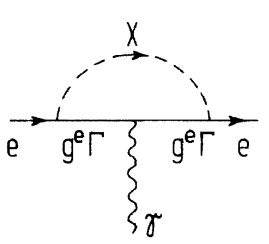$$
G_{\mu}\left(p^{\prime}, p, z_{k}\right)=\Gamma_{i}\left[p^{\prime}\left(1-z_{2}\right)-p z_{3}+m\right]
$$$$
\times \gamma_{\mu}\left[p\left(1-z_{3}\right)-p^{\prime} z_{2}+m\right] \Gamma^{i} \text {. }
$$

(a)

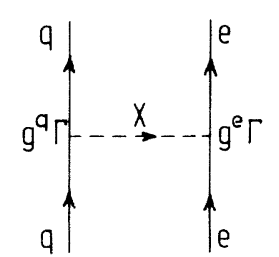

(b)
FIG. 5. Feynman graphs for (a) the electron anomalous magnetic moment, (b) the interaction of the electron with quarks. $m$ is the lepton mass and $\Gamma_{i}$ denotes the Dirac matrices leading to bilinear covariants with definite Lorentz transformation properties. To be specific, we have $i=S$, $P, V$, and $A$ for scalar, pseudoscalar, vector, and axial vector, i.e., $\Gamma_{S}=1, \Gamma_{P}=i \gamma^{5}, \Gamma_{V}=\gamma^{\mu}$, and $\Gamma_{A}=\gamma^{\mu} \gamma^{5}$.

In (7) a term contributing to the charge form factor only has been omitted. To obtain the $g$ factor of a free particle, the expectation value of $\Lambda_{\mu}\left(p^{\prime}, p\right)$ between free spinors $\bar{u}\left(p^{\prime}\right)$ and $u(p)$ has to be evaluated, subsequently performing the limit $p^{\prime} \rightarrow p$, i.e., $q^{2} \rightarrow 0$ for the photon momentum.

The numerator (8) depends on the type of coupling through the matrix $\Gamma_{i}$. By repeated use of the anticommutation relations for the Dirac matrices and of the free Dirac equation (i.e., substituting $\not p \rightarrow m$ when acting to the right and $p^{\prime} \rightarrow m$ to the left) the function can be reduced to the form 


$$
G_{\mu}\left(p^{\prime}, p, z_{k}\right)=m f_{i}\left(z_{k}\right)\left(p_{\mu}^{\prime}+p_{\mu}\right)+m^{2} g_{i}\left(z_{k}\right) \gamma_{\mu} .
$$

Here $f_{i}$ and $g_{i}$ are dimensionless functions of the set of integration variables $z_{k}$, the form of which depends on the commutation properties of the matrix $\Gamma_{i}$. Using the Gordon decomposition of the electromagnetic current

$$
\begin{aligned}
\bar{u}\left(p^{\prime}\right) \gamma^{\mu} u(p)=\bar{u}\left(p^{\prime}\right)[ & \left(p^{\prime}+p\right)^{\mu} / 2 m \\
& \left.+(i / 2 m) \sigma^{\mu v}\left(p^{\prime}-p\right)_{v}\right] u(p),
\end{aligned}
$$

the electron anomaly can be expressed as

$$
a=\frac{1}{2}(g-2)=a_{\mathrm{QED}}+\Delta a_{i}
$$

where $a_{\mathrm{QED}}$ is the standard quantum electrodynamics (QED) radiative correction and $\Delta a_{i}$ is the additional correction due to the new particle of type $i$

$$
\Delta a_{i}=\left(\alpha_{i}^{\mathrm{e}} / 2 \pi\right) K_{i}\left(m_{X} / m\right)
$$

with the integral

$$
K_{i}=\int_{0}^{1} d z_{1} \int_{0}^{1-z_{1}} d z_{2} f_{i}\left(z_{1}, z_{2}\right) /\left[\left(1-z_{1}\right)^{2}+\rho z_{1}\right]
$$

We use the abbreviation $\rho=\left(m_{X} / m\right)^{2}$. For the various types of coupling the explicit form of the function $f_{i}$ is

$$
\begin{aligned}
& f_{S}=-2\left(1+z_{1}\right) z_{2}, \\
& f_{P}=+2\left(1-z_{1}\right) z_{2}, \\
& f_{V}=+4 z_{1} z_{2}, \\
& f_{A}=-4 z_{1}\left(2-z_{2}\right) .
\end{aligned}
$$

The integrals (13) can be solved by elementary methods leading to the following results:

$$
\begin{aligned}
& K_{S}=-\left[\frac{3}{2}-\rho+\frac{1}{2} \rho(\rho-3) \ln \rho-\left(\rho^{2}-5 \rho+4\right) F(\rho)\right], \\
& K_{P}=+\left[\frac{1}{2}+\rho-\frac{1}{2} \rho(\rho-1) \ln \rho+\rho(\rho-3) F(\rho)\right], \\
& K_{V}=+\left[1-2 \rho+\rho(\rho-2) \ln \rho-2\left(\rho^{2}-4 \rho+2\right) F(\rho)\right], \\
& K_{A}=-\left[-9+2 \rho-\left(\rho^{2}-6 \rho+4\right) \ln \rho+2\left(\rho^{2}-8 \rho+14\right) F(\rho)\right],
\end{aligned}
$$

where $F(\rho)$ denotes the function

$$
\begin{aligned}
& F(\rho)=[\rho /(\rho-4)]^{1 / 2} \operatorname{Arth}[(\rho-4) / \rho]^{1 / 2} \text { if } \rho>4, \\
& F(\rho)=[\rho /(4-\rho)]^{1 / 2} \arctan [(4-\rho) / \rho]^{1 / 2} \text { if } \rho<4 .
\end{aligned}
$$

If the mass ratio $m_{X} / m$ becomes very small the coefficients reduce to

$$
\begin{aligned}
& K_{S} \rightarrow-\frac{3}{2}, \text { for } \rho<1 \\
& K_{P} \rightarrow+\frac{1}{2}, \\
& K_{V} \rightarrow+1, \\
& K_{A} \rightarrow 4 \ln \rho \rightarrow-\infty .
\end{aligned}
$$

The value of $K_{V}$ immediately is recognized as Schwinger's result for the anomalous magnetic moment due to the vertex correction of the (massless) photon. In the opposite limit of high mass ratio the coefficients approach zero like

$$
\begin{aligned}
& K_{S} \rightarrow-(1 / \rho) \ln \rho, \text { for } \rho>1 \\
& K_{P} \rightarrow+(1 / \rho) \ln \rho, \\
& K_{V} \rightarrow+\left(\frac{2}{3}\right)(1 / \rho), \\
& K_{A} \rightarrow-\left(\frac{10}{3}\right)(1 / \rho) .
\end{aligned}
$$

Since the expression (15) for the coefficients $K_{i}$ are not very illuminating we have plotted their values against the mass ratio $m_{X} / m$ in Fig. 6. Also indicated in the figure are the values of $m_{X} / m$ for the case of electrons and of muons if one assumes $m_{X}=1.68 \mathrm{MeV}$.

From the known level of agreement between theory and experiment for the anomalous magnetic moment $\left(\Delta a^{\text {expt }}<3 \times 10^{-10}\right.$ for the electron and $\Delta a^{\text {expt }}<1 \times 10^{-8}$ for the muon ${ }^{21}$ ) we deduce upper limits for the coupling constants. For the $X$-boson-electron coupling we find $\alpha_{S}^{\mathrm{e}}<1.1 \times 10^{-8}, \quad \alpha_{P}^{\mathrm{e}}<1.6 \times 10^{-8}, \quad \alpha_{V}^{\mathrm{e}}<4 \times 10^{-8}$, $\alpha_{A}^{\mathrm{e}}<8 \times 10^{-9}$. In the case of the muon the constraints are less severe due to the lower experimental accuracy: $\alpha_{S}^{\mu}<4 \times 10^{-8}, \alpha_{P}^{\mu}<10^{-7}, \alpha_{V}^{\mu}<7 \times 10^{-8}, \alpha_{A}^{\mu}<3 \times 10^{-9}$.

There is one possible loophole in this argument: If there exist several new particles of different coupling type, their contributions to the anomalous magnetic moment may cancel each other, thus allowing for larger values of the coupling constant. We discuss this for the most likely (or better, least unlikely) case of a pseudoscalar particle of mass $m_{X}=1.68 \mathrm{MeV}$ and an additional scalar particle. Using the function $K_{S}(\rho)$ we can deduce the ratio of coupling constants $g_{S} / g_{P}$ required to give $\Delta a_{S}+\Delta a_{P}=0$ and thus invalidate the constraint posed by the $g$-factor measurement. The values of $g_{S} / g_{P}$ as a function of the mass $m_{S}$ are shown in Fig. 7 for the electron and the muon (dashed line) anomalous magnetic moment.

If we assume that the scalar particle has a large mass then also its coupling constant has to be large in order to offset the decrease of the function $K_{S}(\rho)\left(\propto m_{S}^{-2}\right)$. For

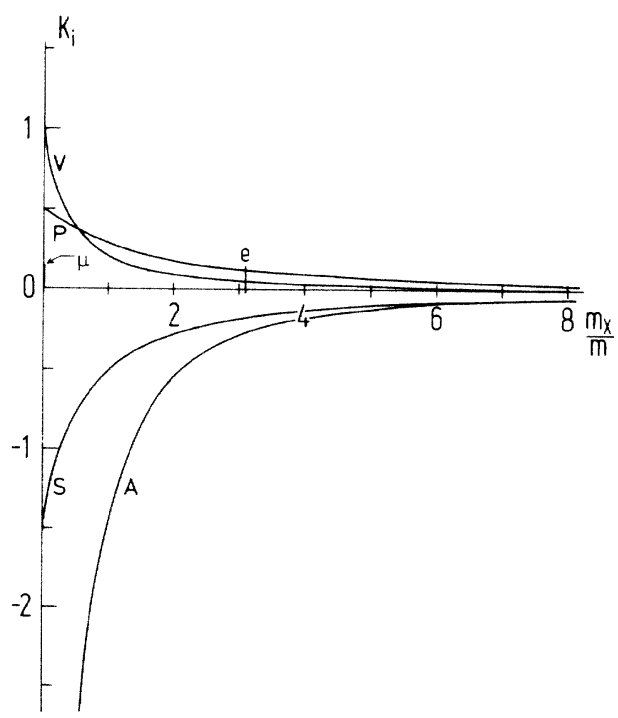

FIG. 6. The coefficients $K_{i}\left(m_{X} / m\right)$ describing the contribution of the postulated new particle to the anomalous magnetic moment of a lepton of mass $m$, cf. Eq. (12). 


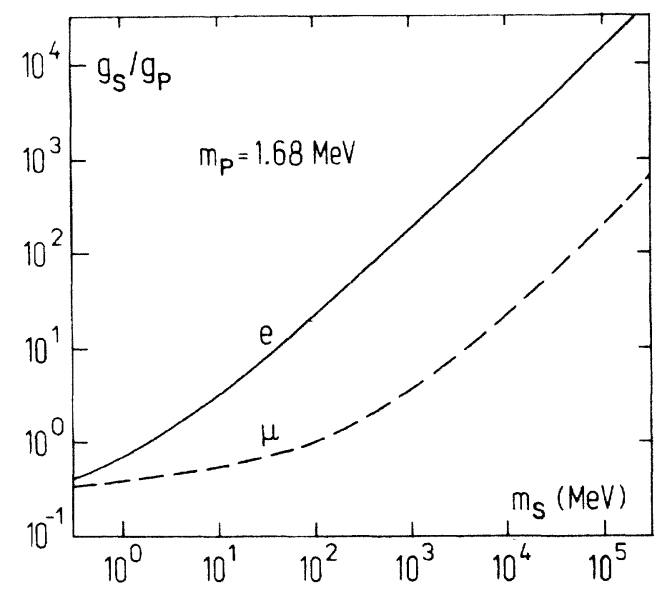

FIG. 7. The ratios of the coupling constants $g_{S}^{\mathrm{e}} / g_{P}^{\mathrm{e}}$ (full line) and $g \xi / g_{F}^{\mu}$ (dashed line) for which the contributions of a scalar particle and pseudoscalar particle of mass $1.68 \mathrm{MeV}$ to the anomalous magnetic moment of the electron (full line) or muon (dashed line) mutually cancel.

example, if $m_{S}=100 \mathrm{GeV}$ then a coupling constant of $g_{S} \simeq 1.4 \times 10^{4} g_{P}$ is required to balance completely the contribution of the pseudoscalar particle to the anomalous magnetic moment of the electron. Thus the introduction of a heavy scalar particle can hardly improve the limit on $\alpha_{P}^{\mathrm{e}}$ obtained earlier if one wants to avoid extremely large coupling constants $\alpha_{S}^{\mathrm{e}}$.

In the case of a light scalar particle, on the other hand, an accurate finetuning of the coupling constants is needed to achieve cancellation of $\Delta a_{S}$ and $\Delta a_{P}$. If the masses are equal, $m_{S}=m_{P}$, a ratio of $g_{P}^{e} / g_{S}^{e} \simeq 1.173$ is required, which has to be kept with an accuracy of

$$
\delta g_{S}^{\mathrm{e}} / g_{S}^{\mathrm{e}}<\left|\left(2 \pi \Delta a_{\text {expt }}^{\mathrm{e}}\right) /\left(\alpha_{S}^{\mathrm{e}} 2 K_{S}\right)\right| \simeq 6 \times 10^{-9} / \alpha_{S}^{\mathrm{e}} .
$$

Independently, for the muon the condition $g_{\mu}^{\mu} / g_{S}^{\mu} \simeq 1.706$ with $\delta g_{S}^{\mu} / g_{S}^{\mu}<2 \times 10^{-8} / \alpha_{S}^{\mu}$ has to be satisfied. While this can be arranged in principle, the condition seems very artificial. Due to the different numerical values for the ratio of coupling constants, electron muon universality would be completely lost in this scheme.

From the upper bounds for $\alpha_{i}^{\mathrm{e}}$ it is possible to derive lower bounds for the lifetime of the $X$ particle against the two-body decay $X \rightarrow \mathrm{e}^{+}+\mathrm{e}^{-}$. For the various types of coupling we find the following expression for the decay width:

$$
\Gamma_{i}=\frac{1}{2} m_{X} \alpha_{i}^{\mathrm{e}} F_{i}(\rho),
$$

where the $F_{i}(\rho)$ are slowly varying functions of the mass ratio $\rho=\left(m_{X} / m\right)^{2}$ :

$$
\begin{aligned}
& F_{S}(\rho)=(1-4 / \rho)^{3 / 2}, \\
& F_{P}(\rho)=(1-4 / \rho)^{1 / 2}, \\
& F_{V}(\rho)=\left(\frac{2}{3}\right)(1-4 / \rho)^{1 / 2}(1+2 / \rho), \\
& F_{A}(\rho)=\left(\frac{2}{3}\right)(1-4 / \rho)^{3 / 2} .
\end{aligned}
$$

The lifetime $\tau_{i}=\hbar / \Gamma_{i}$ is found to be $\tau_{i}=2 \hbar /\left[m_{X} \alpha_{i}^{\mathrm{e}} F_{i}(\rho)\right] \simeq 7.9 \times 10^{-22} \mathrm{~s} /\left[\alpha_{i}^{\mathrm{e}} F_{i}(\rho)\right]$.
For a particle mass of $m_{X}=1.68 \mathrm{MeV}$ the functions $F_{i}(\rho)$ take on a value of $F_{S}=0.5, F_{P}=0.79, F_{V}=0.63$, and $F_{A}=0.33$. Using the limits on $\alpha_{i}^{\mathrm{e}}$ deduced earlier we get $\tau_{S}>1 \times 10^{-13} \mathrm{~s}, \tau_{P}>6 \times 10^{-14} \mathrm{~s}, \tau_{V}>2 \times 10^{-14} \mathrm{~s}$, and $\tau_{A}>3 \times 10^{-13} \mathrm{~s}$.

Finally we want to mention another problem connected with the hypothetical production of an $X$ particle. There have been extensive searches for light, weakly interacting particles decaying into $\mathrm{e}^{+} \mathrm{e}^{-}$pairs. ${ }^{22,23}$ The null result of all these experiments can probably only be understood if one assumes $\tau \leq 10^{-10} \mathrm{~s}$. Then the $X$ particle would have decayed before it could penetrate the heavy shielding necessary in all these experiments. However, for such lifetimes the mechanism described in Sec. II to produce a narrow line will hardly work.

\section{ESTIMATE OF THE LEPTONIC PRODUCTION CROSS SECTION}

In a heavy ion collision light bosons in principle could be created due to their interaction with the atomic electrons, with the moving nuclei, or with the electromagnetic field. We first want to discuss the former mechanism. Its calculation in principle can proceed in complete analogy to that of the process of photon emission. This has been treated for the case of quasimolecular $\mathrm{x}$-ray radiation in heavy ion collisions. A comprehensive formulation using field theoretical methods has been given in Ref. 24. While the creation mechanism itself may be treated in first-order perturbation theory, a detailed knowledge of the dynamics of the many-electron system is required. According to Ref. 24 the number of particles emitted per energy and solid angle interval is

$$
\frac{d^{2} w}{d \Omega d p_{0}}=\frac{p}{16 \pi^{3}}\left(\sum_{\substack{n>F \\ m<F}}\left|H^{n m}\right|^{2}+\left|\sum_{n} H^{n n}\right|^{2}\right) \text {. }
$$

Here $H^{n m}$ denotes the Fourier transform of the timedependent "radiation" matrix element

$$
H^{n m}=g_{i}^{\mathrm{e}} \int_{-\infty}^{\infty} d t e^{i p_{0} t}\left\langle\psi_{n}\left|\Gamma_{i} e^{-i \mathbf{p} \cdot \mathbf{x}}\right| \psi_{m}\right\rangle
$$

The wave functions $\psi_{n}(\mathbf{x}, t)$ are solutions of the timedependent one-electron Dirac equation

$$
i \partial / \partial t \psi_{n}(\mathbf{x}, t)=\left\{\boldsymbol{\alpha} \cdot \mathbf{p}+\beta m+V_{\mathrm{TC}}[\mathbf{R}(t)]\right\} \psi_{n}(\mathbf{x}, t),
$$

where the nuclear motion enters via the two-center potential $V_{\text {TC }}$ which depends on the internuclear distance $\mathbf{R}(t)$. The dynamical wave functions $\psi_{n}(\mathbf{x}, t)$ can be obtained from a basis expansion, in particular by using the adiabatic eigenstates of the two-center quasimolecule as a basis. ${ }^{25}$ Alternatively, a direct numerical integration method can be employed. ${ }^{26}$ The index of $\psi_{n}$ denoted the initial condition (at $t \rightarrow-\infty)$ from which the wave function evolves in time.

When evaluating Eq. (20) the Fermi level $F$ has to be specified. The states of the lower continuum of the Dirac equation are contained in the set $m<F$. The tilde sum in the second term of (20) is defined as 


$$
\widetilde{\sum_{n}}=\frac{1}{2}\left(\sum_{n<F}-\sum_{n>F}\right)
$$

The expression (20) displays great generality: It describes transitions caused by real atomic electrons as well as vacuum fluctuation effects induced by the timedependent nuclear Coulomb potential. In practice, of course, the calculation has to be truncated, concentrating on those channels considered to be most important.

We have not attempted to solve Eqs. (20) and (21) for the process of $X$-boson creation. However, an estimate of the emission intensity can be gained from the already existing calculations of photon emission. In high- $Z$ systems the matrix elements for the various types of coupling are about equal in magnitude. Therefore, very roughly, the cross section for $X$-boson creation should correspond to the cross section for the emission of photons with energy $E_{\gamma}>m_{X}$, multiplied by the ratio of coupling constants

$$
\sigma_{X} \simeq\left(\alpha_{i}^{\mathrm{e}} / \alpha\right) \int_{m_{X}}^{\infty} d E_{\gamma}\left(d \sigma / d E_{\gamma}\right)
$$

As an example, for $\mathrm{Pb}+\mathrm{Pb}$ collisions at 5.9 $\mathrm{MeV} /$ nucleon impact energy the calculated value of $\sigma_{\gamma}\left(E_{\gamma}>m_{X}\right)$ is about $400 \mu \mathrm{b}^{27}$ This is in agreement with measurements of quasimolecular $x$-ray radiation. ${ }^{28}$ Using an upper limit of $\alpha_{i}^{\mathrm{e}}<10^{-8}$ for the coupling constant (see Sec. III). This leads to the estimate $\sigma_{X} \simeq 5 \times 10^{-10} \mathrm{~b}$. This falls short of the required value of $\sigma_{\mathrm{e}^{+}} \simeq 50 \mu \mathrm{b}$ by five orders of magnitude. A similar discrepancy was found in the calculation of Ref. 15.

Although our argument is based on a rough approximation it is hard to imagine a mechanism which enhances the production rate by such a large factor. Therefore, the hypothesis that a light boson created from the atomic electron cloud is responsible for the observed positron line seems to be untenable.

\section{ATOMIC PHYSICS CONSTRAINTS}

If a light new particle which couples to electrons and nuclei existed, it would contribute to the atomic binding energies. These have been calculated and measured with a high precision and no discrepancies have been found. Thus we can deduce, e.g., from the Lamb-shift experiments in hydrogen an upper limit for the product of the $X$-electron and $X$-proton coupling constants: $g_{i}^{\mathrm{p}} g_{i}^{\mathrm{e}}$.

On the level of a quantum field theory one would introduce a quark- $X$ coupling term in the Lagrangian

$$
L_{q, X}=\sum_{f} g_{i}^{f} \bar{\psi}_{f} \Gamma_{i} \psi_{f} \phi_{X},
$$

where $f$ counts the quark flavors. [This interaction corresponds to the Feynman graph in Fig. 5(b).] From this one can derive an effective nucleon- $X$ interaction, in terms of which we will analyze the constraints from atomic physics.

$$
L_{N, X}=\left(g_{i}^{\mathrm{p}} \sum_{\mathrm{p}=1}^{\mathrm{Z}} \bar{\psi}_{\mathrm{p}} \Gamma_{i} \psi_{\mathrm{p}}+g_{i}^{\mathrm{n}} \sum_{\mathrm{n}=1}^{N} \bar{\psi}_{\mathrm{n}} \Gamma_{i} \psi_{\mathrm{n}}\right) \phi_{X} .
$$

The actual calculation of $g_{i}^{\mathrm{p}}$ and $g_{i}^{\mathrm{n}}$ from the $X$-quark coupling is not easy, e.g., for $i=P$ current algebra tech- niques are needed. The effective interaction (25) is sufficient to discuss whether a new particle can be responsible for the positron peak at all. However, the relation between the nucleon- $X$ and the quark- $X$ couplings becomes important if one wants to incorporate the postulated new particle into the framework of quantum field theory.

The nucleons are treated nonrelativistically. For the different $\Gamma_{i}(i=S, P, V, A)$ this leads to the following source terms:

$$
\begin{aligned}
& i=S: \sum_{\mathrm{n}=1}^{N} \bar{\psi}_{\mathrm{n}} \psi_{\mathrm{n}} \rightarrow \sum_{\mathrm{n}=1}^{N} \rho_{\mathrm{n}}=\rho_{N}, \\
& i=P: \sum_{\mathrm{n}=1}^{N} \bar{\psi}_{\mathrm{n}} \gamma_{5} \psi_{\mathrm{n}} \rightarrow 0, \\
& i=V: \sum_{\mathrm{n}=1}^{N} \bar{\psi}_{\mathrm{n}} \gamma_{\mu} \psi_{\mathrm{n}} \rightarrow \sum_{\mathrm{n}=1}^{N} \rho_{\mathrm{n}} \delta_{\mu 0}=\rho_{N} \delta_{\mu 0}, \\
& i=A: \sum_{\mathrm{n}=1}^{N} \bar{\psi}_{\mathrm{n}} \gamma_{0} \gamma_{5} \psi_{\mathrm{n}} \rightarrow 0, \\
& \sum_{\mathrm{n}=1}^{N} \bar{\psi}_{\mathrm{n}} \gamma \gamma_{5} \psi_{\mathrm{n}} \rightarrow \sum_{\mathrm{n}=1}^{N} \rho_{\mathrm{n}}\left(-2 \mathrm{~s}_{n}\right),
\end{aligned}
$$

where $s_{\mathrm{n}}$ denotes the spin of the nth neutron. The proton currents lead to the corresponding results.

The sources (26) lead to an additional Yukawa-type interaction potential of range $1 / m_{X}$. The nature of this interaction depends on $i$. For $i=S$ one gets a scalar potential and for $i=V$ a contribution to the Coulomb potential. For an axial vector $X$ particle the Yukawa potential mediates a spin-spin interaction. For $i=P$ there is no nonrelativistic source and thus in a first approximation we get no constraints for the couplings of a pseudoscalar $\boldsymbol{X}$ particle. The constraints for $g_{i}^{\mathrm{p}}$ and $g_{i}^{\mathrm{n}}$ obtained in the other three cases are quite similar. Therefore we only discuss here the situation for scalar $X$ particles. ${ }^{29}$

(i) The most stringent constraint comes from the Lamb shift in hydrogen. The experiment value ${ }^{30} \Delta E$ $=1057.845 \pm 0.009 \mathrm{MHz}$ agrees with theoretical predictions $^{31}$ to better than $0.03 \mathrm{MHz}$. From this we deduce a limit for the combined coupling constants $g^{\mathrm{e}} g^{\mathrm{p}}<2 \times 10^{-8}$.

(ii) One gets a further constraint for $g^{N}$ $\left[g^{N}=\left(Z g^{\mathrm{p}}+N g^{\mathrm{n}}\right) /(Z+N)\right]$ from the energy levels of heavy atoms. For example the $K_{\alpha}$ transition energy in fermium is not affected significantly only if $g^{N} g^{\mathrm{e}}<10^{-6}$.

(iii) It is also possible to derive upper bounds for $g^{\mu} g^{N}$ from myonic atoms. Although the muon-nucleon interaction is not of immediate interest for our problem we just state for completeness that one gets, e.g., from the analysis of the $5 g_{9 / 2} \rightarrow 4 f_{7 / 2}$ transition energy in $\mu \mathrm{Pb}$, the constraint $g^{\mu} g^{N}<10^{-7}$.

Additional constraints on the coupling strengths can also be obtained from the analysis of, e.g., neutron-nucleus and electron-neutron scattering. We quote some bounds found in the literature for scalar $X$ particles: ${ }^{32}$

(1) Neutron-nucleus scattering leads to $g^{\mathrm{n}}<6 \times 10^{-5}$.

(2) From electron-neutron scattering one infers 
$g^{\mathrm{e}} g^{\mathrm{n}}<3 \times 10^{-8}$.

Finally we want to note that one can also think of a purely electromagnetic production of the $X$ particles. Such a possibility was investigated in Ref. 33 for the case of a pseudoscalar $X$ particle.

\section{BREMSSTRAHLUNG OF (PSEUDO-) SCALAR PARTICLES}

At energies at or below the nuclear Coulomb barrier we expect that the emission of light (scalar or pseudoscalar) particles is mainly caused by the collective deceleration of the colliding nuclei, i.e., by a mechanism of bremsstrahlung-type. To obtain an estimate of the emission probability we have performed a calculation based on the semiclassical approximation.

In accordance with the well established treatment of photon bremsstrahlung ${ }^{34}$ and subthreshold pion production $^{35}$ we seek a solution of the inhomogeneous wave equation

$$
\left(\square+m_{X}^{2}\right) \phi(\mathbf{x}, t)=\rho(\mathbf{x}, t) .
$$

The source term $\rho(\mathbf{x}, t)$, which follows from the interaction Lagrangian discussed in Sec. V, in principle should be determined from the dynamics of the nucleon fields. We will approximate it by two localized density distributions traveling on Rutherford trajectories. Solving (27) with the retarded Green's function, the energy flux in the radiation zone can be calculated. Division by the energy carried by a single particle leads to an expression for the number of particles emitted per energy interval $d p_{0}$ and per solid angle $d \Omega$

$$
\frac{d^{2} w}{d \Omega d p_{0}}=\left(p / 16 \pi^{3}\right)\left|\int d^{4} x e^{i\left(p_{0} t-\mathbf{p} \cdot \mathbf{x}\right)} \rho(\mathbf{x}, t)\right|^{2}
$$

with $p \equiv|\mathbf{p}|$. Note that the emission of electromagnetic bremsstrahlung is determined by a similar formula

$$
\begin{aligned}
\frac{d^{2} w}{d \Omega d p_{0}}= & \left(1 / 4 \pi^{2}\right)\left(1 / p_{0}\right) \\
& \times\left|\mathbf{p} \times \int d^{4} x e^{i\left(p_{0} t-\mathbf{p} \cdot \mathbf{x}\right)} \mathbf{j}(\mathbf{x}, t)\right|^{2},
\end{aligned}
$$

where $\mathbf{j}(\mathbf{x}, t)$ is the electromagnetic current.

To evaluate the Fourier integral in (28) we have to specify the source term $\rho(\mathbf{x}, t)$. In the case of scalar coupling it will be determined by the nucleon density. Assuming full coherence of the nucleon motion in each nucleus, we will approximate

$$
\rho_{S}(\mathbf{x}, t)=\sum_{i=1}^{2} C_{i} \delta^{3}\left[\mathbf{x}-\mathbf{R}_{i}(t)\right],
$$

where the index $i$ enumerates the colliding nuclei. If we further assume for simplicity isospin independence of the interaction, the source strength $C_{i}$ simply is the product of the scalar coupling constant and the nucleon number

$$
C_{i}=g_{S}^{N} A_{i} .
$$

The finite extension (form factor) of the nucleus can easily be incorporated in (30), but this should be unimportant as long as the deBroglie wavelength of the emitted particle is large compared with the nuclear radius. (Note that this condition is satisfied for low energy emission of particles with mass in the $\mathrm{MeV}$ range but not for pions, for which the nuclear extension must be taken into account.) Inserting (30) the emission spectrum (28) is reduced to a one-dimensional integral in time

$$
\begin{aligned}
\left(\frac{d^{2} w}{d \Omega d p_{0}}\right)_{S}= & \left(p / 16 \pi^{3}\right) \\
& \times\left|\int_{-\infty}^{\infty} d t e^{i p_{0} t} \sum_{i=1}^{2} C_{i} e^{-i \mathrm{p} \cdot \mathbf{R}_{i}(t)}\right|^{2} .
\end{aligned}
$$

The construction of a source term in the case of pseudoscalar coupling is less straightforward. The original expression in terms of the nucleon field $\Psi$

$$
\rho_{P}=-i g_{P}^{N} \bar{\Psi} \gamma^{5} \Psi
$$

does not have a classical limit. To obtain an expression that lends itself to interpretation in terms of collective variables, we make use of the long-known approximate equivalence between pseudoscalar coupling and pseudovector derivative coupling. ${ }^{36}$ With the help of the Dirac equation

$$
(p-M) \Psi=i g_{P}^{N} \gamma^{5} \Psi \phi
$$

and its adjoint the expression (33) may be transformed to the form ${ }^{35}$

$$
\rho_{P}=\left(g_{P}^{N} / 2 M\right) \partial_{\mu}\left(\bar{\Psi} \gamma^{5} \gamma^{\mu} \Psi\right)-\left[\left(g_{P}^{N}\right)^{2} / M\right] \bar{\Psi} \Psi \phi .
$$

Here $M$ is the nucleon mass. The last term in (35) is of second order in the coupling constant. It contains a selfinteraction of the boson field and will be neglected in the following discussion. The first term contains a fourdivergence of a pseudovector quantity which is recognized to represent the nucleon spin density. Following Ref. 35 we assume that the spin density is proportional to the number density of nucleons $\rho(x)$, i.e.,

$$
\Psi \gamma^{5} \gamma^{\mu} \Psi=\rho_{P}(x) \sigma^{\mu}(x) \text {. }
$$

The spin vector $\sigma^{\mu}$ is obtained from the spin vector $\sigma^{\mu}=(0, s)$ in the particle restframe via a Lorentz boost with velocity $v$

$$
\sigma^{\mu}=\left(-\mathbf{s} \cdot \mathbf{v}, \mathbf{s}+\left[(\gamma-1) / v^{2}\right](\mathbf{s} \cdot \mathbf{v}) \mathbf{v}\right) .
$$

Inserting (35) and (37) into (28) after an integration by parts and neglect of the surface term leads to ${ }^{35}$

$$
\left\lfloor\frac{d^{2} w}{d \Omega d p_{0}}\right\rfloor_{P}=\left(p / 16 \pi^{3}\right)\left|\int d^{4} x e^{i\left(p_{0} t-p \cdot x\right)}\left(g_{N}^{P} / 2 M\right) \sum_{\alpha} \rho_{\alpha}(x) \sigma_{\alpha}^{\mu} p_{\mu}\right|^{2}
$$


where the sum runs over all the nucleons. We now again approximate the source by two localized pointlike nuclei moving on classical trajectories [Eq. (30)]

$$
\left[\frac{d^{2} w}{d \Omega d p_{0}}\right]_{P}=\left(p / 16 \pi^{3}\right)\left|\int_{-\infty}^{\infty} d t e^{i p_{0} t} \sum_{i=1}^{2}\left(C_{i} / 2 M\right) \mathbf{s}_{i} \cdot \mathbf{p} e^{-i \mathbf{p} \cdot \mathbf{R}_{i}(t)}\right|^{2} .
$$

To further simplify the expression here we have replaced $\sigma^{\mu} p_{\mu}$ by the nonrelativistic approximation $\mathbf{s} \cdot \mathbf{p}$. Note that $\mathbf{s}$ is the average spin per nucleon.

To solve the Fourier integrals in Eqs. (32) or (39) the trajectory $\mathbf{R}(t)$ has to be specified. At energies below the Coulomb barrier the nuclei move on Rutherford hyperbolae. We use the convenient parameter representation for the internuclear distance coordinate $R=R_{1}-R_{2}$

$$
\begin{aligned}
& R(w)=a(\epsilon \cosh w+1), \\
& X(w)=a(\cosh w+\epsilon), \\
& Y(w)=a\left(\epsilon^{2}-1\right)^{1 / 2} \sinh w, \\
& Z(w)=0, \\
& t(w)=\left(a / v_{0}\right)(\epsilon \sinh w+w),
\end{aligned}
$$

where $a=Z_{1} Z_{2} / 2 E_{\text {c.m. }}$ is half the distance of closest ap- proach for head-on collisions, $\epsilon=\left[1+(b / a)^{2}\right]^{1 / 2}$ is the eccentricity of the hyperbola, and $v_{0}$ is the asymptotic relative nuclear velocity. The trajectories of the two colliding nuclei (in the center of mass frame) are given by

$$
\begin{aligned}
& \mathbf{R}_{1}(t)=\left[m_{2} /\left(m_{1}+m_{2}\right)\right] \mathbf{R}(t), \\
& \mathbf{R}_{2}(t)=-\left[m_{1} /\left(m_{1}+m_{2}\right)\right] \mathbf{R}(t) .
\end{aligned}
$$

As in the case of photon bremsstrahlung it is advantageous to perform a Taylor expansion of the exponential function

$$
e^{-i \mathbf{p} \cdot \mathbf{R}_{i}(t)}=1-i \mathbf{p} \cdot \mathbf{R}_{i}(t)-\frac{1}{2}\left[\mathbf{p} \cdot \mathbf{R}_{i}(t)\right]^{2}+\cdots .
$$

This corresponds to a multipole expansion of the radiation field. $^{34}$ It is quite sufficient to retain only the lowest order nonvanishing term. Up to second order the integral in (32) for scalar bremsstrahlung reads

$$
\int_{-\infty}^{\infty} d t e^{i p_{0} t} \sum_{i=1}^{2} C_{i} e^{-i \mathbf{p} \cdot \mathbf{R}_{i}(t)}=\int_{-\infty}^{\infty} d t e^{i p_{0} t}\left(M-i p n_{k} D_{k}-\frac{1}{2} p^{2} n_{k} n_{l} Q_{k l}+\cdots\right)
$$

with the monopole, dipole, and quadrupole moments

$$
\begin{aligned}
& M=C_{1}+C_{2}, \\
& D_{k}=\mu\left(C_{1} / m_{1}-C_{2} / m_{2}\right) R_{k}, \\
& Q_{k l}=\mu^{2}\left(C_{1} / m_{1}^{2}+C_{2} / m_{2}^{2}\right) R_{k} R_{l} .
\end{aligned}
$$

Here $\mu=m_{1} m_{2} /\left(m_{1}+m_{2}\right)$ is the nuclear reduced mass and we have introduced the normal vector $\mathbf{n}=\mathbf{p} / p$. Since the source strength according to (31) is assumed to be constant in time, no monopole radiation is emitted. Furthermore, if the factors $C_{i}$ are proportional to the nuclear mass, the dipole moment vanishes. (Note that in the electromagnetic case this complete cancellation occurs only if both nuclei have the same charge to mass ratio $Z_{i} / A_{i}$.) Therefore the scalar bremsstrahlung will be mainly of quadrupole-type. For completeness, however, we will also include the dipole term in the following discussion. Using the trajectory (4) the Fourier integrals entering (43) can be solved explicitly. Here we will content ourselves in deriving the emission probabilities integrated over solid angle. The angular averages of the particle momentum unit vector are

$$
\begin{aligned}
& \left\langle n_{i} n_{j}\right\rangle=\frac{1}{3} \delta_{i j}, \\
& \left\langle n_{i} n_{j} n_{k} n_{l}\right\rangle=\frac{1}{15}\left(\delta_{i j} \delta_{k l}+\delta_{i k} \delta_{j l}+\delta_{i l} \delta_{j k}\right) .
\end{aligned}
$$

Inserting (43) into (32) and using (40) leads to the emission spectrum

$$
\begin{aligned}
\left(\frac{d w}{d p_{0}}\right)_{S}=\frac{p^{3}}{4 \pi^{2}} & {\left[\left(\frac{1}{3}\right)\left(\left|D_{X}\right|^{2}+\left|D_{Y}\right|^{2}\right)\right.} \\
& +\left(p^{2} / 60\right)\left(3\left|Q_{X X}\right|^{2}+3\left|Q_{Y Y}\right|^{2}\right. \\
& \left.\left.+4\left|Q_{X Y}\right|^{2}+2 Q_{X X} Q_{Y Y}\right)\right]
\end{aligned}
$$

where the Fourier transforms $D_{k}\left(p_{0}\right)$ and $Q_{k l}\left(p_{0}\right)$ of the multipole moments defined in (44) enter. These integrals can be solved analytically in terms of modified Bessel functions. The final result is

$$
\left[\frac{d w}{d p_{0}}\right]_{S}=(2 / \pi)\left(p^{3} / p_{0}^{2}\right) a^{2}\left[F_{1} h_{1}+\left(p / p_{0}\right)^{2} v^{2} F_{2} h_{2}\right]
$$


with the abbreviations

$$
\begin{aligned}
& F_{1}=(1 / 4 \pi) \mu^{2}\left(C_{1} / m_{1}-C_{2} / m_{2}\right)^{2}, \\
& F_{2}=(1 / 4 \pi) \mu^{4}\left(C_{1} / m_{1}^{2}+C_{2} / m_{2}^{2}\right)^{2} .
\end{aligned}
$$

If the $C_{i}$ are proportional to the nuclear mass [Eq. (31)] these multipole factors reduce to

$$
\begin{aligned}
& F_{1}=0, \\
& F_{2}=\left[\left(g_{S}^{N}\right)^{2} / 4 \pi\right] A_{R}^{2},
\end{aligned}
$$

where $A_{R}=A_{1} A_{2} /\left(A_{1}+A_{2}\right)$ is the reduced nucleon number. The shape of the emission spectrum is determined by the functions

$$
\begin{aligned}
& h_{1}=\left(\frac{2}{3}\right) e^{-\pi v}\left\{\left[\left(\epsilon^{2}-1\right) / \epsilon^{2}\right] K^{2}+K^{\prime 2}\right\}, \\
& \begin{aligned}
& h_{2}=\left(\frac{2}{15}\right) e^{-\pi v}\left\{\left(1 / \epsilon^{4}\right)\left[3 \epsilon^{4}-4 \epsilon^{2}+4+4 v^{2}\left(\epsilon^{2}-1\right)^{3}\right] K^{2}\right. \\
&+\left.4 v\left[\left(\epsilon^{2}-1\right) / \epsilon^{3}\right]\left(4-3 \epsilon^{2}\right) K K^{\prime}+\left(\epsilon^{2}-1\right) / \epsilon^{2}\left[4 v^{2}\left(\epsilon^{2}-1\right)+4\right] K^{\prime 2}\right\} .
\end{aligned}
\end{aligned}
$$

$v$ denotes the ratio between the particle energy $p_{0}$ and the characteristic Fourier frequency $\omega_{0}$ of the collision

$$
v=p_{0} / \omega_{0}=p_{0} a / v_{0}
$$

Finally, $K$ denotes the MacDonald function of imaginary order

$$
K \equiv K_{i v}(\epsilon v)=\frac{1}{2} \int_{-\infty}^{\infty} d \eta e^{-\epsilon v \cosh \eta+i v \eta}
$$

and $K^{\prime}$ its derivative with respect to the argument. Except for powers of $p / p_{0}$ which approach unity in the limit of vanishing rest mass, Eq. (46) is very similar to (but not identical with) the corresponding result for electromagnetic bremsstrahlung (cf. Ref. 34, Eq. 9).

To obtain an expression for pseudoscalar bremsstrahlung the Fourier integral of Eq. (39) has to be solved. Expanding the exponential function to first order gives

$$
\int_{-\infty}^{\infty} d t e^{i p_{0} t} \frac{1}{2 M} \sum_{i=1}^{2} C_{i} \mathbf{s}_{i} \cdot \mathbf{p} e^{-i \mathbf{p} \cdot \mathbf{R}_{i}(t)}=\int_{-\infty}^{\infty} d t e^{i p_{0} t} \frac{p}{2 M}\left(n_{k} \widetilde{D}_{k}-i p n_{k} n_{l} \widetilde{Q}_{k l}+\cdots\right)
$$

with the multipole moments

$$
\begin{aligned}
& \widetilde{D}_{k}=\left(C_{1} \mathbf{s}_{1}+C_{2} \mathbf{s}_{2}\right)_{k}, \\
& Q_{k l}=\mu\left[\left(C_{1} / m_{1}\right) \mathbf{s}_{1}-\left(C_{2} / m_{2}\right) \mathbf{s}_{2}\right]_{k} R_{l} .
\end{aligned}
$$

Equation (52) can be evaluated further only if an assumption on the time dependence of the nuclear spin is made. We will make the (perhaps unwarranted) assumption that the vectors $s_{i}$ are constant in time. Then no dipole radiation arises. If (39) is integrated over solid angle $d \Omega$ and averaged over the spin directions we obtain

$$
\left(\frac{d w}{d p_{0}}\right]_{P}=(2 / \pi)\left(p^{3} / p_{0}^{2}\right) a^{2}(p / 2 M)^{2}\left(\frac{1}{3}\right) G_{1} h_{1},
$$

where $h_{1}$ is the function defined in (49). The magnitude of the factor $G_{1}$ depends on the relative orientation of the nuclear spins. It takes its maximum value

$$
\begin{aligned}
G_{1}= & (1 / 4 \pi) \mu^{2}\left[\left(C_{1} / m_{1}\right) s_{1}+\left(C_{2} / m_{2}\right) s_{2}\right]^{2} \\
& \rightarrow\left[\left(g_{P}^{N}\right)^{2} / 4 \pi\right] A_{R}^{2} 4 s^{2}
\end{aligned}
$$

if $\mathbf{s}_{1}$ and $\mathbf{s}_{2}$ are completely anticorrelated and vanishes in the case of complete correlation (parallel spins).

The differential emission cross section $d \sigma / d E_{X}$ for scalar or pseudoscalar particles is obtained by integrating Eqs. (47) and (54) over impact parameter b. As an exam- ple the results are shown in Fig. 8 for a collision of $U+U$ at $6 \mathrm{MeV} /$ nucleon bombarding energy and assuming a particle mass of $m_{X}=1.68 \mathrm{MeV}$. The spectra exhibit broad maxima at $E_{X} \simeq 600 \mathrm{keV}$ and decay exponentially at high energies. At low kinetic energy $E_{X}$ the intensity is suppressed due to the powers of $p$ entering Eqs. (47) and (54). Note that this would be different in the case of vanishing rest mass of the emitted particle (e.g., photon bremsstrahlung) due to the identity $p=p_{0}$. In the limit $E_{X} \rightarrow \infty$ the falloff constant is well described by the characteristic Fourier frequency $\omega_{0}$ of the trajectory divided by $2 \pi$, i.e., $d \sigma / d E_{X} \sim \exp \left(-E_{X} / E_{0}\right) \quad$ with $E_{0}=\hbar \omega_{0} / 2 \pi=\hbar v_{0} / 2 \pi a=420 \mathrm{keV}$.

The total emission cross section is found by numerical integration of (47) and (54)

$$
\begin{aligned}
& \sigma_{X}^{S}=6.4 \times 10^{-3} \mathrm{~b} \times\left(g_{S}^{N}\right)^{2} / 4 \pi \\
& \sigma_{X}^{P}=5.3 \times 10^{-7} \mathrm{~b} \times\left(g_{P}^{N}\right)^{2} / 4 \pi \times(\text { spin } / \text { nucleon })^{2} .
\end{aligned}
$$

Note that the numbers in Eq. (56), as well as the differential cross sections given in Fig. 8, still must be multiplied by the coupling constants $\left(g_{i}^{N}\right)^{2} / 4 \pi$. The pseudoscalar result furthermore has to be multiplied by the square of the average spin per nucleon. The suppression of $\sigma_{X}^{P}$ relative to $\sigma_{X}^{S}$ is mainly due to the factor $(p / 2 M)^{2}$ in Eq. (54) since the relevant energies are small compared to the nucleon mass $\boldsymbol{M}$. 


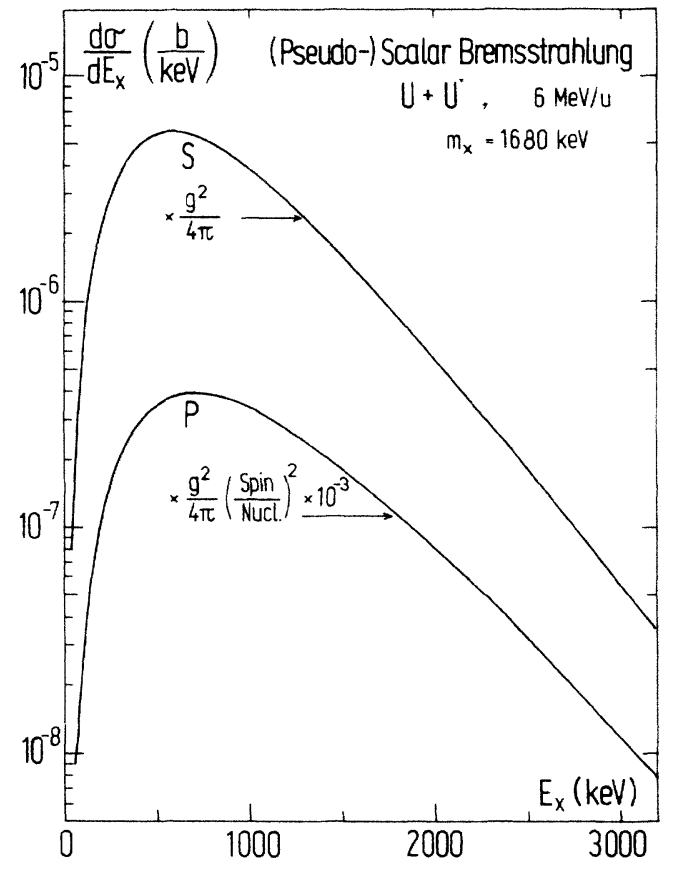

FIG. 8. Differential cross section for scalar (upper curve) and pseudoscalar (lower curve) particles produced by nuclear bremsstrahlung in a $6 \mathrm{MeV} /$ nucleon $U+U$ collision calculated within the semiclassical approximation.

Now we will try to answer the question, whether the creation of a new particle through the discussed bremsstrahlung-type mechanism can be invoked to explain the measured positron lines.

First we note that the spectra $d \sigma / d E_{X}$ displayed in Fig. 8 certainly will not lead, without further assumptions, to a narrow positron line. Due to the suppression of $d \sigma / d E_{X}$ at small energies the positron energy distribution will be very broad, as demonstrated in Fig. 3. Even in the case of particles with a long lifetime, i.e., when most of the fast emitted particles decay outside the detector, no narrow structure emerges. This argument alone should be sufficient to rule out the described bremsstrahlung mechanism. Nevertheless, let us consider the possibility that the expressions (47) and (54) for some reason underestimate the emission spectrum at low particle momentum. Then the argument can be based on the total cross sections given in Eq. (56).

Taking into account the lifetime argument of Sec. III, to explain the positron production cross section we have to identify $\sigma_{\mathrm{e}^{+}}^{\text {expt }} \simeq \sigma_{X}\left(1-e^{-T / \tau}\right)$. The experimental cross section $^{3,4}$ is of the order $\sigma_{\mathrm{e}^{+}}^{\text {ext }} \geq 50 \mu \mathrm{b}$. The effective "detector escape time" $T$ is typically of the order $10^{-10} \mathrm{~s}$. If, as required from the linewidth argument, $\tau \gg 10^{-10} \mathrm{~s}$, we have to require $\sigma_{\mathrm{e}^{+}}^{\text {ext }} \simeq \sigma_{X} T / \tau$.

Let us first discuss the case of scalar particles. Using the approximate expression for the lifetime, $\tau \simeq 10^{-21} \mathrm{~s} / \alpha^{\mathrm{e}}$ [see Eq. (19)], we arrive at the condition

$$
\alpha_{S}^{N} \alpha_{S}^{\mathrm{e}} \geq(50 \mu \mathrm{b} / 6400 \mu \mathrm{b})\left(10^{-21} \mathrm{~s} / 10^{-10} \mathrm{~s}\right) \simeq 10^{-13}
$$

This conclusion is independent of the assumption of long lifetime. In the opposite limiting case $\left(\tau<10^{-10} \mathrm{~s}\right)$ we would have found separately $\alpha_{S}^{\mathrm{e}}>10^{-11}$ (from the lifetime formula) and $\alpha_{S}^{N} \geq 10^{-2}$ (from requiring $\sigma_{\mathrm{e}^{+}}^{\text {expt }}=\sigma_{X}^{S}$ ) which gives the same result for $\alpha_{S}^{N} \alpha_{S}^{e}$.

For this product of coupling constants, however, in Sec. $\mathrm{V}$ an upper limit of $\alpha_{S}^{N} \alpha_{S}^{\mathrm{e}} \leq 10^{-18}$ has been found from the atomic physics data. Clearly these constraints are not compatible.

If we turn to the case of pseudoscalar particles, we have not deduced an upper limit for the product of coupling constants $\alpha_{P}^{N} \alpha_{P}^{\mathrm{e}}$. The very small value of $\sigma_{X}^{P}$, however, is sufficient to rule out this possibility. Using $\sigma_{\mathrm{e}^{+}}^{\text {expt }}=\sigma_{X}^{P}$, i.e., omitting the additional reduction factor due to long lifetimes, we have $\alpha_{P}^{N} \geq 10^{4}$ if the average spin per nucleon is (quite arbitrarily) taken as 0.1 . Clearly such a large coupling constant is not meaningful.

\section{ELECTRON-POSITRON SCATTERING}

The most obvious way to produce a new boson of the type discussed in this paper is to look for a resonance in low-energy electron-positron scattering. This should be found at a total center-of-mass energy $E^{\prime}=m_{X}$ or, assuming electrons at rest as a target, a laboratory positron energy $E_{R}=m_{X}^{2} / 2 m-m$. Assuming a mass $m_{X}=1.68 \mathrm{MeV}$ this leads to a kinetic impact energy $E=1.74 \mathrm{MeV}$. In the following we will write the contribution of a scalar or pseudoscalar boson to the scattering cross section and address the question whether a resonance effect can be observed experimentally.

In lowest order the scattering of electrons and positrons is determined by the Feynman graph for direct onephoton exchange plus the annihilation graph. The Bhabha formula (see, e.g., Ref. 37) takes into account the squares of the two corresponding invariant amplitudes and the interference term. If in addition an $X$ boson is exchanged, the amplitude consists of the terms

$$
\begin{aligned}
\left|M_{f i}\right|^{2}=\mid & M_{f i}(\gamma, \text { dir })+M_{f i}(\gamma, \text { anni }) \\
& +M_{f i}(X, \operatorname{dir})+\left.M_{f i}(X, \text { anni })\right|^{2} .
\end{aligned}
$$

In general this leads to seven additional terms contributing to the scattering cross section. In the special case of a pseudoscalar particle the two $X$ - $\gamma$-interference terms for direct and for annihilation scattering vanish identically. The resonance occurs at timelike momenta of the virtual boson, i.e., in the annihilation graph. Therefore we will calculate the contributions resulting from

$$
\mid\left. M_{f i}(X, \text { anni })\right|^{2}
$$

and

$$
2 \operatorname{Re} M_{f i}^{*}(\gamma, \operatorname{dir}) M_{f i}(X, \text { anni }) .
$$

In the laboratory system (electrons initially at rest) the differential scattering cross section, averaged over spin polarization, for the resonance in the annihilation channel is (note that in this section we omit the superscript e as no misunderstanding is possible) 


$$
\left.\int \frac{d \sigma}{d \Omega}\right\rfloor_{P}(X, \text { anni })=\left(\alpha_{P}^{2} / m^{2}\right) \frac{\cos \theta}{\left(1-b^{2} \cos ^{2} \theta\right)^{2}} \frac{m^{2}}{\left(E-E_{R}\right)^{2}+\left(m_{X} \Gamma / 2 m\right)^{2}} .
$$

Here $b^{2}=(E-m) /(E+m)$ (which is the squared center of mass velocity). The expression for the case of scalar particles is very similar

$$
\left[\frac{d \sigma}{d \Omega}\right]_{S}(X, \text { anni })=\left(\frac{d \sigma}{d \Omega}\right]_{P}(X, \text { anni }) b^{2},
$$

where, of course, $\alpha_{P}$ has to be replaced by $\alpha_{S}$. In the derivation of (59) the boson was given a complex mass $m_{X}-i \Gamma / 2$ to account for its finite lifetime. The angular dependence of (59) is purely kinematic, in the c.m. frame the cross section is isotropic.

The largest interference term between photon and massive boson exchange takes the form

$$
\int \frac{d \sigma}{d \Omega} \int_{P}(X, \text { anni; } \gamma, \operatorname{dir})=-2\left(\alpha \alpha_{P} / m^{2}\right) \frac{\cos \theta}{\sin ^{2} \theta\left(1-b^{2} \cos ^{2} \theta\right)} \frac{1}{\beta^{2} \gamma} \frac{m\left(E-E_{R}\right)}{\left(E-E_{R}\right)^{2}+\left(m_{X} \Gamma / 2 m\right)^{2}},
$$

and

$$
\begin{aligned}
\left.\int \frac{d \sigma}{d \Omega}\right]_{S}(X, \text { anni; } \gamma, \text { dir })= & -2\left(\alpha \alpha_{P} / m^{2}\right) \frac{\cos \theta}{\sin ^{2} \theta\left(1-b^{2} \cos ^{2} \theta\right)} \frac{1}{\beta^{2} \gamma^{2}(\gamma+1)} \\
& \times\left(\gamma^{2}-3 \gamma-2 \frac{1+b^{2} \cos ^{2} \theta}{1-b^{2} \cos ^{2} \theta}\right) \frac{m\left(E-E_{R}\right)}{\left(E-E_{R}\right)^{2}+\left(m_{X} \Gamma / 2 m\right)^{2}} .
\end{aligned}
$$

Here $\beta=|p| / E$ and $\gamma=\left(1-\beta^{2}\right)^{-1 / 2}$. Integration of (59) over $d \Omega$ leads to the total resonance cross section

$\sigma_{P}(X$, anni $)=\left(\alpha_{P}^{2} / m^{2}\right) \frac{\pi}{2}(\gamma+1) \frac{m^{2}}{\left(E-E_{R}\right)^{2}+\left(m_{X} \Gamma / 2 m\right)^{2}}$,

and a similar expression for the scalar case.

Obviously, the problem with these results lies in the smallness of the coupling constant $\alpha_{i}$ which suppresses the resonance cross section with respect to ordinary scattering via photon exchange. When considering Eqs. (59) - (61) we have to keep in mind that the width $\Gamma$ is of the order $\alpha_{i} m$ (cf. Sec. III) which is very small.

Exactly at its peak value the resonance cross section would exceed the Coulomb background by several orders of magnitude. In any realistic experiment, however, the cross section has to be averaged over the energy spread $\Delta E$ of the beam. Assuming, for simplicity, that the energy distribution has a Lorentzian shape we have to average

$$
\langle\sigma(E)\rangle=(\Delta E / 2 \pi) \int_{-\infty}^{\infty} d \epsilon \frac{1}{(\epsilon-E)^{2}+(\Delta E / 2)^{2}} \sigma(\epsilon) .
$$

If the spread $\Delta E$ is large compared to the intrinsic width $\Gamma$ this procedure simply leads to the replacement of $m_{X} \Gamma / 2 m$ by $\Delta E / 2$ in the resonance and interference denominators. In addition, the resonance terms are multiplied by the factor $\Delta E /\left(m_{X} \Gamma / m\right)$. The maximum value of the averaged resonance cross section is then

$$
\left\langle\sigma_{P}(X, \mathrm{anni})\right\rangle_{\max }=\left(\alpha_{P} / m^{2}\right) 2 \pi[\rho /(\rho-4)]^{1 / 2}(m / \Delta E),
$$

where $\rho=\left(m_{X} / m\right)^{2}$. The same expression also holds for the scalar case.

We see that the photon, interference, and resonance cross section are of the orders $\alpha^{2}, \alpha \alpha_{i}$, and $\alpha_{i}(m / \Delta E)$, respectively. According to the argument of Sec. III based on the electron anomalous magnetic moment, the coupling constant $\alpha_{i}$ should be smaller than about $10^{-8}$. Using this value the relative intensity of the resonance compared to Bhabha scattering can be calculated in dependence of the energy resolution $\Delta E$. For an angle of, e.g., $\theta=30^{\circ}$, both contributions become equal if $\Delta E \simeq 0.2 \mathrm{keV}$. Going to larger scattering angles this value can be increased by up to a factor of 3 at the expense of the scattering cross section. Since in a precision experiment already a small deviation from the Bhabha cross section would be conspicuous, according to this estimate a measurement of the excitation function of $\mathrm{e}^{+}-\mathrm{e}^{-}$collisions in the appropriate, quite narrow, energy range may be experimentally feasible. Such an experiment is presently under preparation. ${ }^{38}$ This should help to decide on the existence of the postulated new particle, unless its coupling to the electron-positron field is much weaker than the allowed upper limit $\alpha_{\mathrm{e}} \leq 10^{-8}$.

If the coupling constant is much smaller than the deduced upper limit the experiment possibly can take advantage of the finite traveling distance of the created particle. This is of the order

$$
\Delta l=\hbar \beta_{X} \gamma_{X} / \Gamma_{i}=\hbar / m \alpha_{i} \simeq 4 \times 10^{-11} \mathrm{~cm} / \alpha_{i} .
$$

If $\alpha_{i}$ is sufficiently small the decay of the resonant particle is spatially separated from the background elastic scattering which can be used to distinguish between both processes using a suitable detector geometry. 


\section{CONCLUSION}

We have systematically investigated the possibility that the positron line structure observed in the GSI experiments are caused by the two-body decay $X \rightarrow \mathrm{e}^{+}+\mathrm{e}^{-}$of a previously unknown neutral boson with mass $m_{X} \simeq 1.7$ $\mathrm{MeV}$. Assuming that the hypothetical particle is coupled to matter fields by the standard linear interaction $g_{i} \bar{\psi} \Gamma_{i} \psi \phi$ with $i=S, P, V, A$ we have shown that any attempt at a quantitative description of the experimental data is seriously inconsistent with other well established experimental facts.

In particular, the precision data from $(g-2)$ experiments for the electron and muon, from Lamb shift and atomic $K_{\alpha}$-transition measurements, put such stringent limits on the magnitude of the admissible coupling constants $g_{i}$ that the predicted cross section for production of the hypothetical particle in heavy-ion collisions falls short of the observed line intensity by more than four orders of magnitude. We are convinced that this is far outside the uncertainty of our estimates. Furthermore the predicted particle energy distribution does not give rise to a narrow structure in the positron spectrum. This would require a mechanism predominantly producing slow particles. Also the two-photon coupling based on the chiral anomaly, $(\alpha / f) \mathbf{E} \cdot \mathbf{H} \phi$, with reasonable values of the decay constant $f$, has been shown in a separate study ${ }^{33}$ to give cross sections that are too small by many orders of magnitude.

As already discussed in Sec. III it is, in principle, possible to circumvent the limit derived from the $(g-2)$ experiment by introducing pairs of scalar and pseudoscalar particles, with finely tuned coupling constant to produce cancellation of their contributions to the anomalous magnetic moment. In this case the limit can be relaxed to $\alpha_{\mathrm{e}} \leq 3 \times 10^{-6}$ which is set by the hyperfine splitting in positronium. ${ }^{39}$ This still rules out the leptonic production mechanism as source of the hypothetical particle.

Does this conclusively rule out a new particle as the source of the observed positron lines? The answer depends on one's taste to some extent. While linearly coupled interactions can be safely ruled out on the basis of the arguments presented here, nonlinear interactions cannot be discounted so easily. Two types of nonlinearity come to mind: either a nonlinear interaction with the matter fields, e.g., $g \phi(\bar{\psi} \Gamma \psi)^{n}$, most likely the nucleon field, or a nonlinear coupling to the strong electromagnetic fields which exist in the heavy-ion collision and might influence the coupling strength to the matter fields, $g=g(\mathbf{E}, \mathbf{H})$.

All these nonlinear interactions would give rise to a nonrenormalizable theory and must, therefore, be considered as effective interactions describing the low-energy properties of a more complicated microscopic theory. Especially, a speculation that the structure of nuclear matter is radically changed above a certain total nucleon number $A$, or above a critical electric field strength $E_{\mathrm{cr}}$, is tempting. ${ }^{40}$ Such a structural change could have particularly strong consequences on the coupling strength of a pseudoscalar particle, as has been suggested in Ref. 41. Other possible mechanisms could involve a change in the vacuum structure of a Higgs field. ${ }^{42}$ As long as the consequences, and the viability, of such exotic mechanisms have not been fully explored, the "particle scenario" for the origin of the positron lines cannot be ruled out. However, in view of our results, and in the absence of other experimental facts supporting the onset of dramatic nonlinear effects, it must be considered as highly speculative.

\section{ACKNOWLEDGMENTS}

We thank the members of the experimental groups at GSI for stimulating discussions, in particular $\mathbf{H}$. Backe, K. Bethge, H. Bokemeyer, T. Cowan, J. S. Greenburg, P. Kienle, Ch. Kozhuharov, D. Schwalm, J. Schweppe, and K. E. Stiebing. We are also grateful to G. Soff, GSI for his collaboration. This work was supported by GSI and Bundesministerium für Forschung und Technologie.
*Permanent address: Institut für Theoretische Physik, J. W. Goethe-Universität, Frankfurt, Federal Republic of Germany. 1J. Schweppe, A. Gruppe, K. Bethge, H. Bokemeyer, T. Cowan, H. Folger, J. S. Greenberg, H. Grein, S. Ito, R. Schulé, D. Schwalm, K. E. Stiebing, N. Trautmann, P. Vincent, and $\mathbf{M}$. Waldschmidt, Phys. Rev. Lett. 51, 2261 (1983).

${ }^{2}$ M. Clemente, E. Berdermann, P. Kienle, H. Tsertos, W. Wagner, C. Kozhuharov, F. Bosch, and W. Koenig, Phys. Lett. 137B, 41 (1984).

${ }^{3}$ T. Cowan, H. Backe, M. Begemann, K. Bethge, H. Bokemeyer, H. Folger, J. S. Greenberg, H. Grein, A. Gruppe, Y. Kido, M. Klüver, D. Schwalm, J. Schweppe, K. E. Stiebing, N. Trautmann, and P. Vincent, Phys. Rev. Lett. 54, 1761 (1985).

${ }^{4}$ H. Tsertos, E. Berdermann, F. Bosch, M. Clemente, P. Kienle, W. Koenig, C. Kozhuharov, and W. Wagner, Phys. Lett. 162B, 372 (1985).

$5 \mathrm{~J}$. Schweppe (unpublished).

6P. Kienle, Gesellschaft für Schwerionenforschung Report
GSI-85-31, 1985.

${ }^{7}$ B. Müller, J. Rafelski, and W. Greiner, Z. Phys. 257, 62 (1972); 257, 183 (1972); Ya. B. Zeldovich and V. S. Popov, Usp. Fiz. Nauk 105, 403 (1971) [Sov. Phys. Usp. 14, 673 (1972)]; J. Reinhardt and W. Greiner, Rep. Prog. Phys. 40, 219 (1977).

8J. Reinhardt, B. Müller, and W. Greiner, Phys. Rev. A 24, 103 (1981).

${ }^{9}$ J. Reinhardt, U. Müller, B. Müller, and W. Greiner, Z. Phys. A 303, 173 (1981).

10J. Rafelski, B. Müller, and W. Greiner, Z. Phys. A 285, 49 (1978).

${ }^{11}$ U. Heinz, U. Müller, J. Reinhardt, B. Müller, and W. Greiner, Ann. Phys. (N.Y.) 158, 476 (1984).

${ }^{12}$ M. Seiwert, W. Greiner, and W. T. Pinkston, J. Phys. G 11, L21 (1985).

${ }^{13}$ T. Tomoda and H. A. Weidenmüller, Phys. Rev. C 28, 739 (1983).

${ }^{14}$ A. Schäfer, J. Reinhardt, B. Müller, W. Greiner, and G. Soff, 
J. Phys. G 11, L69 (1985).

${ }^{15}$ A. B. Balantekin, C. Bottcher, M. R. Strayer, and S. J. Lee, Phys. Rev. Lett. 55, 461 (1985).

16R. D. Peccei and H. Quinn, Phys. Rev. Lett. 38, 1440 (1977); Phys. Rev. D 16, 1791 (1977).

${ }^{17}$ S. Weinberg, Phys. Rev. Lett. 40, 223 (1978); F. Wilczek, ibid. 40, 279 (1978).

${ }^{18}$ M. S. Alam et al., Phys. Rev. D 27, 1665 (1983); B. Niczyporuk et al., Z. Phys. C 17, 197 (1983).

${ }^{19}$ R. Hagedorn, Relativistic Kinematics (Benjamin, New York, Amsterdam, 1963)

20J. D. Bjorken and S. D. Drell, Relativistic Quantum Mechanics (McGraw-Hill, New York, 1964).

${ }^{21}$ T. Kinoshita and J. Sapirstein, New Developments in $Q E D$, Atomic Physics 9 (World-Scientific, Singapore, 1984).

${ }^{22}$ T. W. Donnelly, S. J. Freedman, R. S. Lytel, R. D. Peccei, and M. Schwarz, Phys. Rev. D 18, 1607 (1978); F. P. Calaprice, R. W. Dunford, R. T. Kouzes, M. Miller, A. Hallin, M. Schneider, and D. Schrieber, ibid. 20, 2708 (1979); P. F. Jacques, M. Kalelkar, P. A. Miller, R. J. Plano, P. Stamer, E. B. Bruckner, E. L. Koller, S. Taylor, C. Baltay, H. French, M. Hibbs, R. Hylton, K. Shastri, and A. Vogel, ibid. 21, 1206 (1980); H. Faissner, E. Frenzel, W. Heinigs, A. Preussger, D. Samm, and U. Samm, Phys. Lett. 96B, 201 (1980); F. Bergsma et al., Phys. Lett. 157B, 458 (1985).

${ }^{23}$ D. Kohler, B. A. Watson, and J. A. Becker, Phys. Rev. Lett. 33, 1628 (1974).

${ }^{24}$ J. Kirsch, B. Müller, and W. Greiner, Z. Naturforsch. 35a, 151 (1983).

${ }^{25}$ G. Soff, J. Reinhardt, B. Müller, and W. Greiner, Z. Phys. A 294, 137 (1980).

${ }^{26}$ U. Becker, N. Grün, and W. Scheid, J. Phys. B 16, 1967
(1983); C. Boettcher and M. R. Strayer, Phys. Rev. Lett. 54, 669 (1985).

27J. Kirsch, W. Betz, J. Reinhardt, B. Müller, and W. Greiner, Z. Phys. A 292, 227 (1979).

${ }^{28}$ W. E. Meyerhof, D. L. Clark, Ch. Stoller, E. Morenzoni, W. Wölfli, F. Folkmann, P. Vincent, P. H. Mokler, and P. Armbruster, Phys. Lett. 70A, 303 (1979); K. E. Stiebing, H. Schmidt-Böcking, W. Schadt, K. Bethge, R. Schuch, P. H. Mokler, F. Bosch, D. Liesen, S. Hagmann, and P. Vincent; Z. Phys. A 319, 239 (1984).

${ }^{29}$ We thank Dr. G. Soff (GSI) for performing these calculations.

30S. M. Lundeen and F. M. Pipkin, Phys. Rev. Lett. 46, 232 (1981).

${ }^{31}$ P. J. Mohr, Phys. Rev. Lett. 34, 1050 (1975).

${ }^{32}$ R. Barbieri and T. E. O. Ericson, Phys. Lett. 57B, 270 (1975).

${ }^{33}$ B. Müller and J. Rafelski (unpublished).

34J. Reinhardt, G. Soff, and W. Greiner, Z. Phys. A 276, 285 (1976).

${ }^{35}$ D. Vasak, B. Müller, and W. Greiner, Phys. Scr. 22, 25 (1980).

36J. M. Berger, L. L. Foldy, and R. K. Osborn, Phys. Rev. 87, 1061 (1952).

37J. M. Jauch and F. Rohrlich, The Theory of Photons and Electrons (Springer, New York, 1976).

${ }^{38} \mathrm{~K}$. Erb, private communication.

39J. Reinhardt (unpublished).

${ }^{40}$ D. Vasak and W. Greiner (unpublished).

${ }^{41}$ B. Müller and J. Rafelski, in Proceedings of the Second International Conference on Nucleus-Nucleus Collisions, Visby, Sweden, 1985.

${ }^{42}$ A. Schäfer, B. Müller, and W. Greiner, Phys. Lett. 149B, 455 (1984). 